This item was submitted to Loughborough's Research Repository by the author.

Items in Figshare are protected by copyright, with all rights reserved, unless otherwise indicated.

\title{
On fracture mode partition theories
}

PLEASE CITE THE PUBLISHED VERSION

http://dx.doi.org/10.1016/j.commatsci.2011.03.021

PUBLISHER

(c) Elsevier

VERSION

AM (Accepted Manuscript)

LICENCE

CC BY-NC-ND 4.0

REPOSITORY RECORD

Wang, Simon, and Liangliang Guan. 2011. "On Fracture Mode Partition Theories". figshare. https://hdl.handle.net/2134/9010. 
This item was submitted to Loughborough's Institutional Repository (https://dspace.lboro.ac.uk/) by the author and is made available under the following Creative Commons Licence conditions.

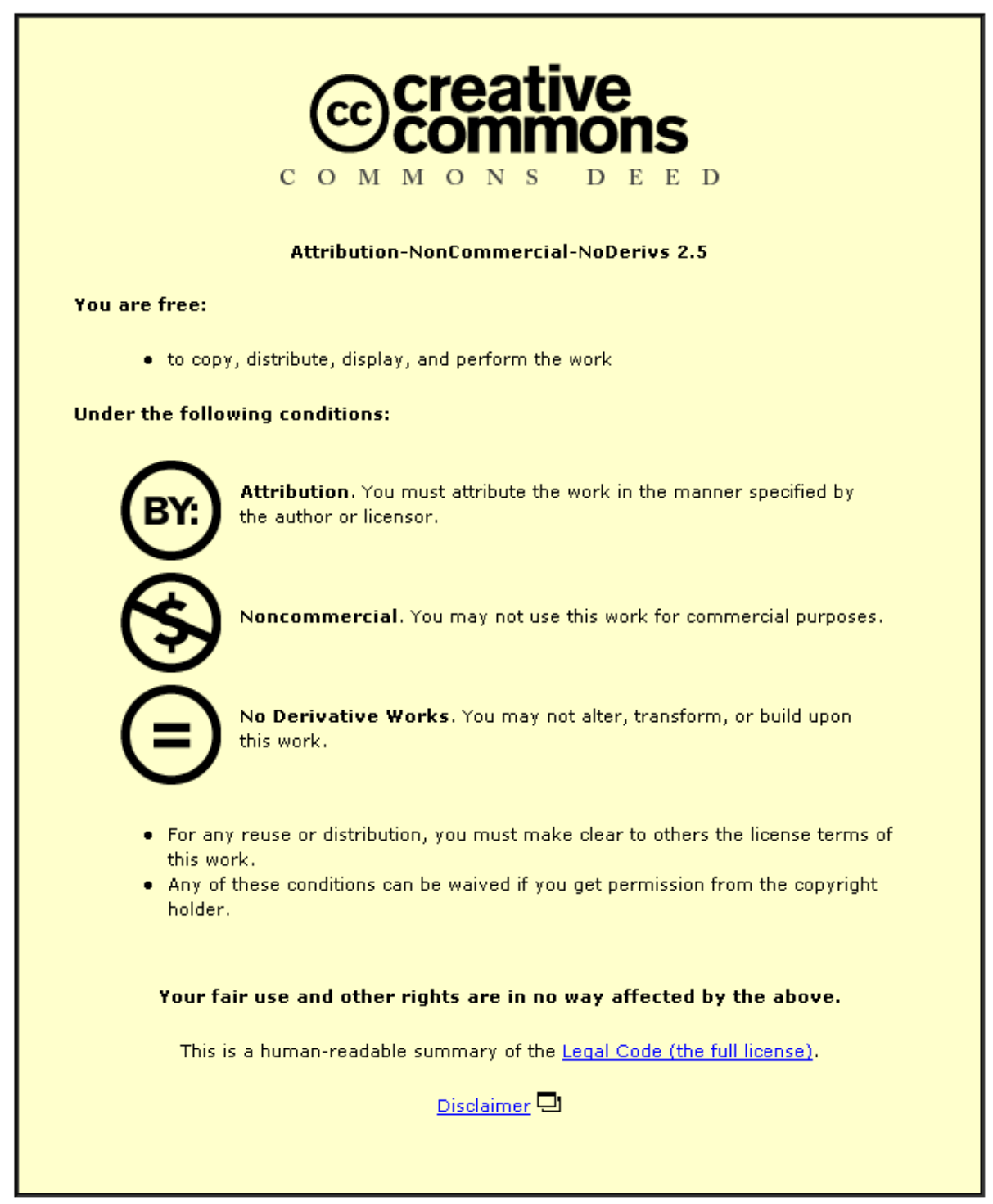

For the full text of this licence, please go to: http://creativecommons.org/licenses/by-nc-nd/2.5/ 


\section{On Fracture Mode Partition Theories}

\section{S. Wang and L. Guan}

\section{S.Wang@loboro.ac.uk}

Department of Aeronautical and Automotive Engineering

University of Loughborough, Loughborough, LE11 3TU, UK

Wang and Harvey (2009 and 2010), and Wang and Guan (2010) have developed fracture mode partition theories for one-dimensional fractures in beams and plates based on both classical and shear deformable beam and plate theories. This paper presents comparisons between different theories and numerical simulations to validate the developed theories.

Keywords: Fracture, Energy release rate, Fracture mode partition

\section{Introduction}

Recently, references [1-5] have developed fracture mode partition theories for one-dimensional fractures in beams and plates based on both classical and shear deformable beam and plate theories. Numerical investigations are presented in this paper to validate these theories using finite element methods in conjunction with interface springs and crack closure technique [6,7]. Section 2 gives a brief summary of the theories in [1-5]. Section 3 presents numerical investigations and conclusions are drawn in Section 4.

\section{Fracture Mode Partitions}

Figure 1 shows a double cantilever beam plate under tip bending moments $\mathrm{M}_{1}$ and $\mathrm{M}_{2}$. The geometry dimensions are also shown in the figure.

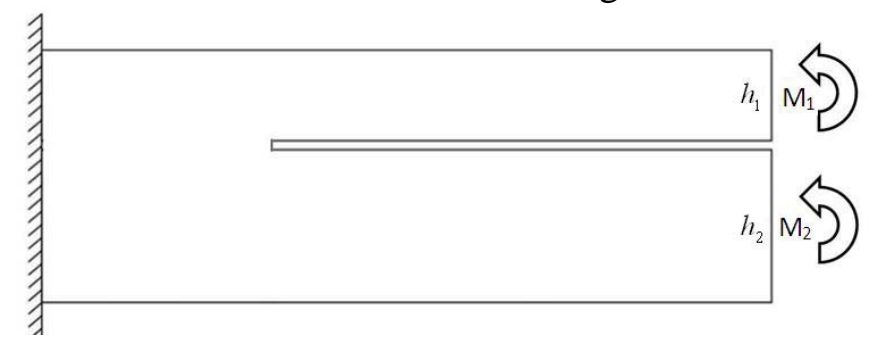

Figure 1: A Double cantilever beam plate

Based on either Euler beam theory or Timoshenko beam theory, the strain energy release rate can be calculated as

$$
\begin{aligned}
& G=\left[M_{1}^{2} / I_{1}+M_{2}^{2} / I_{2}-\left(M_{1}+M_{2}\right)^{2} / I\right] /(2 b E) \\
& =\left\{\begin{array}{ll}
M_{1} & M_{2}
\end{array}\right\}^{T}\left[\begin{array}{cc}
1 / I_{1}-1 / I & -1 / I \\
-1 / I & 1 / I_{2}-1 / I
\end{array}\right]\left\{\begin{array}{l}
M_{1} \\
M_{2}
\end{array}\right\} /(2 b E)
\end{aligned}
$$

(1)

where $b$ is the third dimension of the beam plate. It is noted that no contact is assumed in obtaining the above equation (1).
Now, consider the relative shearing and opening displacements at the cracked interface a small $d a$ distance behind the crack tip. The relative shearing displacement in Timoshenko beam theory is calculated as

$D_{s h}=\left(u_{1 B}+h_{1} / 2 \psi_{1 d a}\right)-\left(u_{2 B}-h_{2} / 2 \psi_{2 d a}\right)$

where

$\psi_{1,2 d a}=M_{1,2} d a /\left(E I_{1,2}\right)+\psi_{1,2 B}$

In the above two expressions, $u$ and $\psi$ represent the mid-plane axial displacement and cross sectional rotations, respectively. The subscript $B$ represents the crack tip position whilst the subscript $d a$ represents the position a small $d a$ distance behind the crack tip. When rigid interface is assumed, the equality of axial displacements at the crack tip interface requires

$u_{1 B}+h_{1} / 2 \psi_{1 B}=u_{2 B}-h_{2} / 2 \psi_{2 B}$

Substituting equations (3) and (4) into equation (2) gives

$$
D_{\text {sh }}=\left[\left(h_{1} M_{1} / I_{1}+h_{2} M_{2} / I_{2}\right) d a\right] /(2 E)
$$

When using Euler beam theory, the cross sectional rotation becomes $\psi=V^{(1)}$ which is the first order derivative of deflection. Obviously, in Euler beam theory, the same expression as equation (5) is obtained. Therefore, letting $D_{\text {sh }}=0$ gives a relationship between the two bending moments as $M_{2}=\left(h_{2} / h_{1}\right)^{2} M_{1}$ for pure mode I, which can be written in vector form as

$\phi_{I}=\left\{\begin{array}{l}1 \\ \theta\end{array}\right\}$

where $\theta=-\gamma^{2}$ and $\gamma=h_{2} / h_{1}$. The pure mode II $\phi_{I I}$ orthogonal to $\phi_{I}$ can be determined using the orthogonal condition with respect to the coefficient matrix of the quadratic form (1). That is, 
$\phi_{I I}=\left\{\begin{array}{l}1 \\ \beta\end{array}\right\}$

In equation (7) $\beta=\gamma^{2}(3+\gamma) /(1+3 \gamma)$ and this pure mode II $\phi_{I I}$ corresponds to zero normal interface stress at the crack tip. This pair of pure modes is designated as $(\theta, \beta)$ pair. Although $(\theta, \beta)$ is a pure pair in both Euler and Timoshenko beam theories, there is subtle difference between the Euler $(\theta, \beta)$ pair and Timoshenko $(\theta, \beta)$ pair, which is revealed below.

The relative opening displacement at the cracked interface a small da distance behind the crack tip in Timoshenko beam theory is determined as

$D_{o p}=\left(\psi_{1 B}-\psi_{2 B}\right) d a$

It can be shown that $\psi_{1 B}=\psi_{2 B}$ in $\beta$ mode leading to $D_{o p}=0$. Therefore, the $\beta$ pure mode II in

Timoshenko beam theory corresponds to both zero crack tip opening force and displacement.

The relative opening displacement in Euler beam theory is determined as

$D_{o p}=\left(M_{1 B} / I_{1}-M_{2 B} / I_{2}\right) d a^{2} /(2 E)$

which leads to a pure mode II

$\phi_{I I}^{\prime}=\left\{\begin{array}{c}1 \\ \beta^{\prime}\end{array}\right\}$

where $\beta^{\prime}=\gamma^{3}$. It is seen that this $\beta^{\prime}$ pure mode II is different from the $\beta$ pure mode II. Similarly, The pure mode I $\phi_{I}^{\prime}$ orthogonal to $\phi_{I I}^{\prime}$ can be determined using the orthogonal condition with respect to the coefficient matrix of quadratic form (1). That is,

$\phi_{I}^{\prime}=\left\{\begin{array}{c}1 \\ \theta^{\prime}\end{array}\right\}$

where $\theta^{\prime}=-1$. Again, this $\theta^{\prime}$ pure mode I is different from the $\theta$ pure mode I.

Now, it can be concluded that there are two pairs of orthogonal pure modes in Euler beam theory and one pair of pure modes in Timoshenko beam theory. In fact, it can be understood that there are also two pairs of pure modes in Timoshenko beam theory, but they coincide to each other. This difference between the two beam theories has fundamental consequence in mixed mode partitions.

By using the $(\theta, \beta)$ pair, $\mathrm{M}_{1}$ and $\mathrm{M}_{2}$ in a general mixed mode can be partitioned as
$\left\{\begin{array}{l}M_{1} \\ M_{2}\end{array}\right\}=\alpha_{I}\left\{\begin{array}{l}1 \\ \theta\end{array}\right\}+\alpha_{I I}\left\{\begin{array}{l}1 \\ \beta\end{array}\right\}=\alpha_{I} \phi_{I}+\alpha_{I I} \phi_{I I}$

where $\alpha_{I}$ and $\alpha_{I I}$ are partition coefficients and can be determined from equation (12). Substituting equation (12) into equation (1) yields

$G=\alpha_{I}^{2} G_{\phi_{I}}+\alpha_{I I}^{2} G_{\phi_{I I}}$

where

$G_{\phi_{I}}=24 \gamma /\left[b^{2} h_{1}^{3} E(1+\gamma)\right]$

and

$G_{\phi_{I I}}=6\left[1+\beta^{2} / \gamma^{3}-(1+\beta)^{2} /(1+\gamma)^{3}\right] /\left(b^{2} h_{1}^{3} E\right)$

In Timoshenko beam theory, the two pairs of pure modes coincide with each other as mentioned above. That is, both the crack tip shearing force and shearing displacement are zero for Timoshenko beam $\theta$ pure mode I whilst both the crack tip opening force and opening displacement are zero for Timoshenko beam

$\beta$ pure mode II. Therefore, there is no any interaction between the $(\theta, \beta)$ pair in Timoshenko beam theory and the strain energy release rate in equation (13) is simply partitioned as

$G_{I}=\alpha_{I}^{2} G_{\phi_{I}}$ and $G_{I I}=\alpha_{I I}^{2} G_{\phi_{I I}}$

However, when using Euler beam theory, there are interactions between the Euler $(\theta, \beta)$ pair. The interactions arise from crack tip opening and shearing forces in $\theta$ mode doing work on crack tip relative opening and shearing displacements in $\beta$ mode. Details on the interaction can be found in References $[3,4]$. Here, a brief consideration is given. By using crack closure technique and equations (1) and (9), the crack opening force in Euler beam $\theta$ mode can be obtained as

$F_{n B \theta} d a=8 \gamma^{2} /(1+\gamma)^{2}$

By using equations (17) and (9), the work done by $F_{n B \theta}$ on $D_{o p \beta}$ is calculated as

$\Delta G_{\phi_{I} \phi_{I I}}=3(\gamma-1)(1+3 \gamma) G_{\phi_{I}}$

$D_{o p \beta}$ is the crack tip opening displacement in Euler beam $\beta$ mode. Since $(\theta, \beta)$ pair is an orthogonal pair, the work done by the crack tip shearing force of $\theta$ mode on the crack tip shearing displacement of $\beta$ mode must be of the same magnitude as given in equation (18) but with an opposite sign. Consequently, the strain energy release rate in equation (13) is partitioned in Euler beam theory as

$G_{I}=\alpha_{I}^{2} G_{\phi_{I}}+\alpha_{I} \alpha_{I I} \Delta G_{\phi_{I} \phi_{I I}}$

and

$G_{I I}=\alpha_{I I}^{2} G_{\phi_{I I}}-\alpha_{I} \alpha_{I I} \Delta G_{\phi_{I} \phi_{I I}}$ 
Numerical validations are given in next section.

\section{Numerical Investigations}

In this Section, numerical investigations are carried out to validate the above theories. In all the investigations, the Young's modulus E is taken as a unit value, the Poisson's ratio $v$ is set to be 0.3 and the shear modulus $G_{13}$ is $1 / 2.6$. In the first test case, $h_{1}+h_{2}=2, b=1$ and $\gamma=2$. The moment $\mathrm{M}_{1}$ on upper beam is fixed at 1 , and the moment $\mathrm{M}_{2}$ on lower beam changes from -10 to 10 . Strain energy release rates $G_{I}$ and $G_{I I}$ and the partitions are presented in Figures 2 and 3. The numerical results consist of three sets of FEM results based on Euler beam (EB), Timoshenko beam (TB) and 2D plane stress theories (2D), respectively. There are three set of analytical results. They are the present mode partition theories based on Euler beam theory (EBA), Timoshenko beam theory (TBA) and Hutchinson and Suo theory (SHA) [8], respectively. Since the present analytical results based Euler and Timoshenko beam theories are almost identical to their counterpart FEM predictions (EB and TB), EBA and TBA curves are not plotted in Figures 2 and 3 for the sake of clearness. Note that the Euler beam FEM predictions (EB) are calculated by using Timoshenko beam FEM with a large shear modulus since direct use of Euler beam theory with interface point springs leads to incompatibility at the interface of lower and upper beams. It is seen that negative $G_{I}$ and $G_{I I}$ in Euler beam theory occur indicating the corresponding crack tip forces do negative work when closing the crack. Although Hutchinson and Suo theory [8] gives the same total strain energy release rate $G$ as those from the present two beam theories, its partitions are different from both of them and in between them. This is expected since the total $G$ in [8] is calculated based on beam theory while the mode partition is based on stress intensity factors which are $2 \mathrm{D}$ elasticity quantities. This explains that Hutchinson and Suo theory [8] agrees very well with 2D FEM results as shown in Figures 2 and 3.

It is seen from Figure 3 that Euler (EB) $G_{I}\left(\right.$ or $\left.G_{I I}\right)$ is larger (or smaller) than Timoshenko (TB) $G_{I}\left(\right.$ or $G_{I I}$ ) if $-4=\theta<M_{2}<\beta=20 / 7$ due to the interaction term in equation (18) which causes a energy flow from mode II to mode I. In the rest of the region, Euler (EB) $G_{I}$ (or $G_{I I}$ ) is smaller (or larger) than Timoshenko (TB) $G_{I}$ (or $G_{I I}$ ) due to energy flow from mode I to mode II. From this observation, it is reasonable to argue that the Euler and Timoshenko beam theories provide either an upper bound or a lower bound for strain energy release rate partitions, respectively. Therefore, it is expected that the average of them may give a good prediction in comparison with the $2 \mathrm{D}$ plane stress partition results. That is, the averaged partition rule gives

$$
G_{I}=\alpha_{I}^{2} G_{\phi_{I}}+1 / 2 \alpha_{I} \alpha_{I I} \Delta G_{\phi_{I} \phi_{I I}}
$$

and

$$
G_{I I}=\alpha_{I I}^{2} G_{\phi_{I I}}-1 / 2 \alpha_{I} \alpha_{I I} \Delta G_{\phi_{I} \phi_{I I}}
$$

AVA curves in Figures 2 and 3 come from this averaged partition rule. It is seen that there is a good agreement between AVA and 2D FEM results.

Finally, note that the present partition theory based on Timoshenko beam theory predicts that crack tip running contact occurs when $M_{2} \geq \beta=20 / 7$ leading to a pure mode II region within $\beta \leq M_{2} \leq \gamma^{3}$. Both Timoshenko and 2D FEM simulations have confirmed this prediction which is not considered in this paper due to space limit.

The second and third investigation cases, i.e., $h_{1}+h_{2}=2, b=1$ and $\gamma=3,4$ are carried out to investigate the effect of $\gamma$ on the theories. The results are presented in Figures 4 and 5 for case 2, and Figures 6 and 7 for case 3, respectively. Again, similar observations can be made. However, it is noted that the comparison between present AVA and 2D FEM results departs away in the negative $G_{I}$ and $G_{I I}$ regions.

The final test is the case 4 , i.e., $h_{1}+h_{2}=6, b=1$ and $\gamma=2$ is considered to study the effect of the total thickness on the present theories. Results are shown in Figures 8 and 9. A good performance of the theories is again observed. 


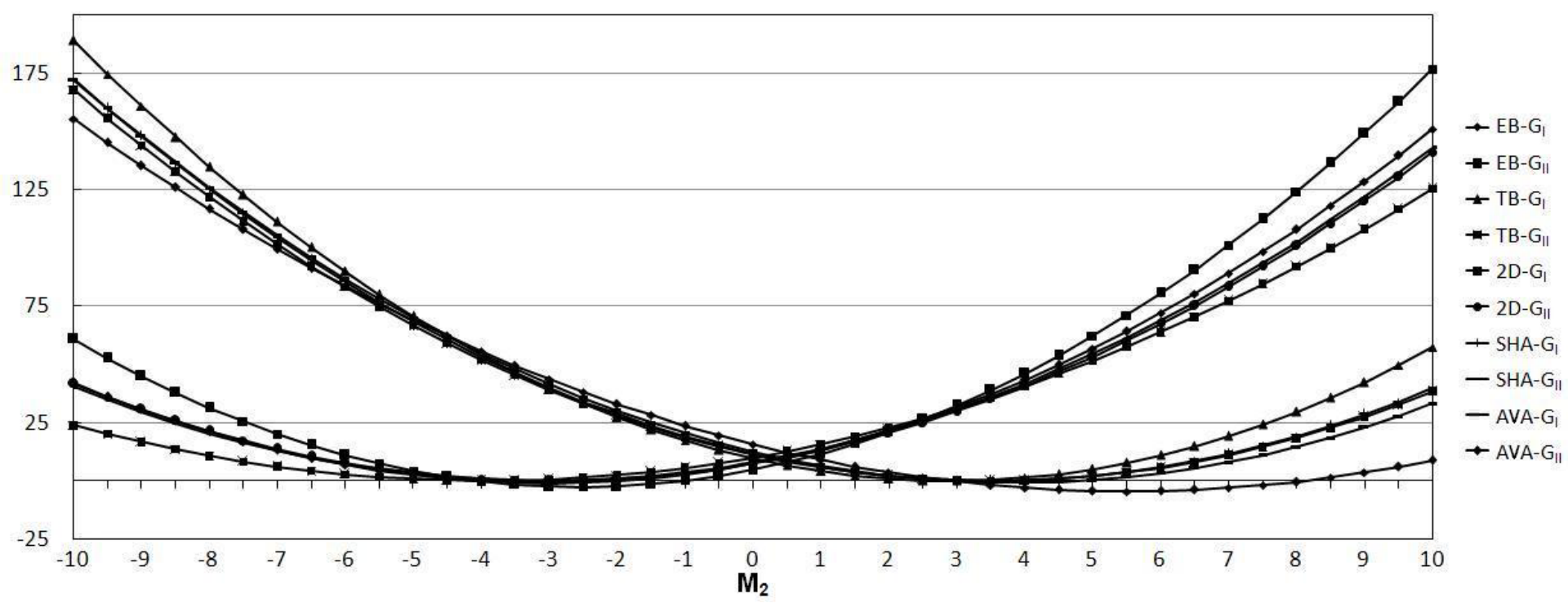

Figure 2: Fracture mode partitions in terms of $G_{I}$ and $G_{I I}$ for case $1, h_{1}+h_{2}=2, b=1$ and $\gamma=2$.

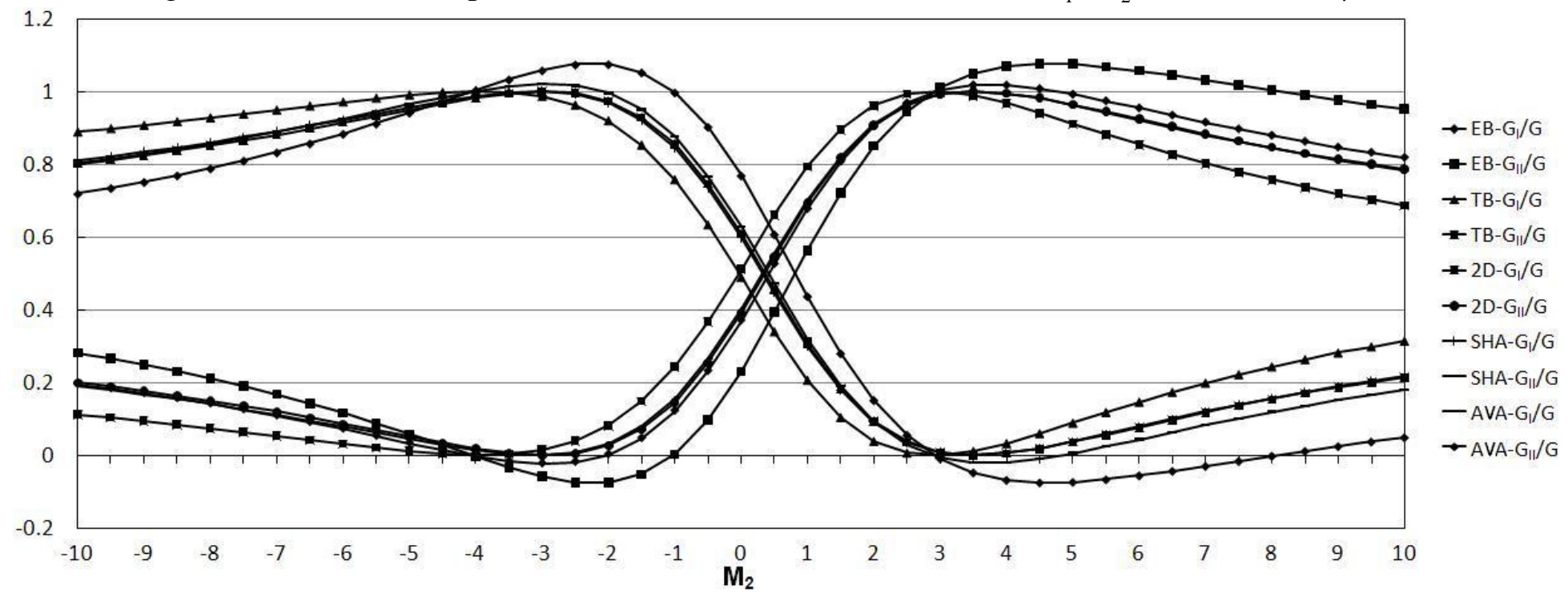

Figure 3: Fracture mode partitions in terms of $G_{I} / G$ and $G_{I I} / G$ for case $1, h_{1}+h_{2}=2, b=1$ and $\gamma=2$. 


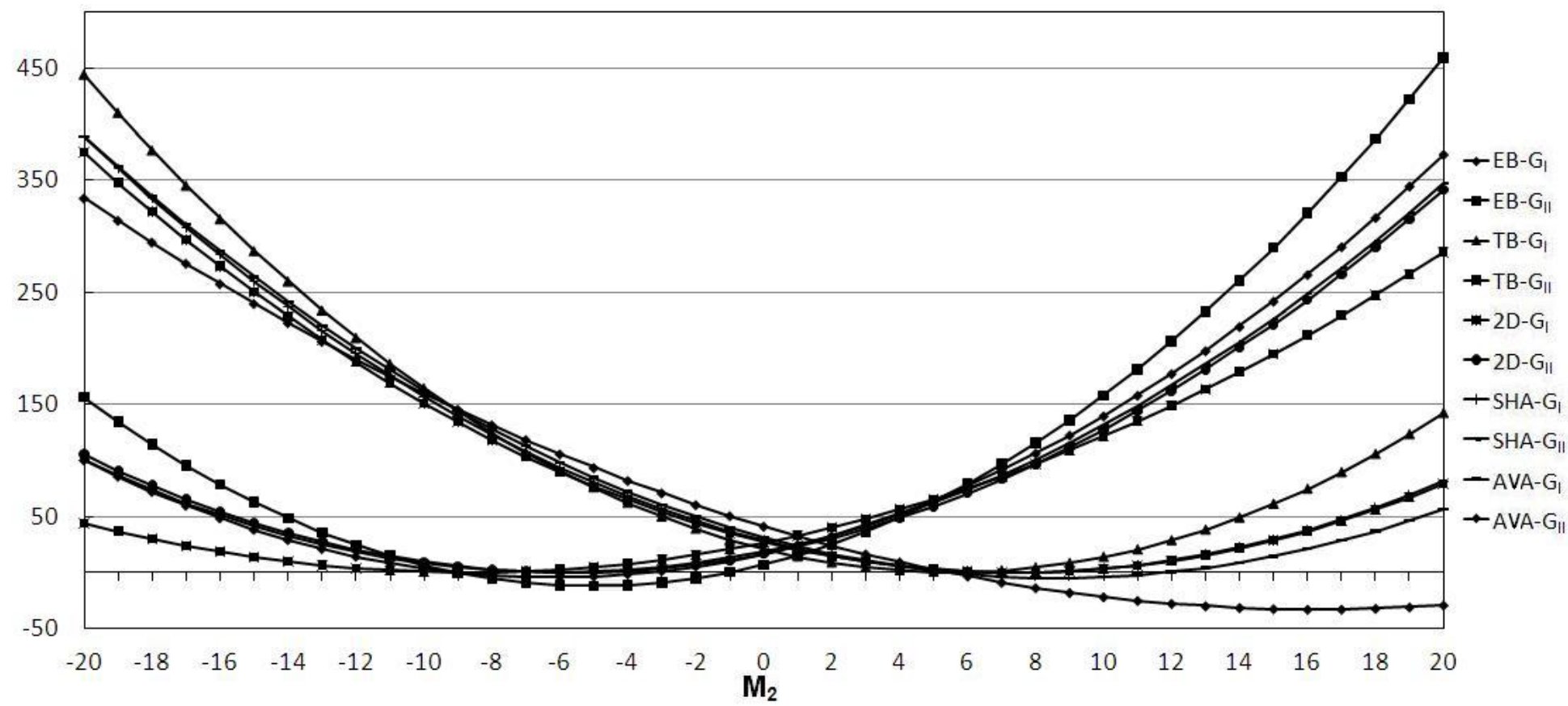

Figure 4: Fracture mode partitions in terms of $G_{I}$ and $G_{I I}$ for case $2, h_{1}+h_{2}=2, b=1$ and $\gamma=3$.

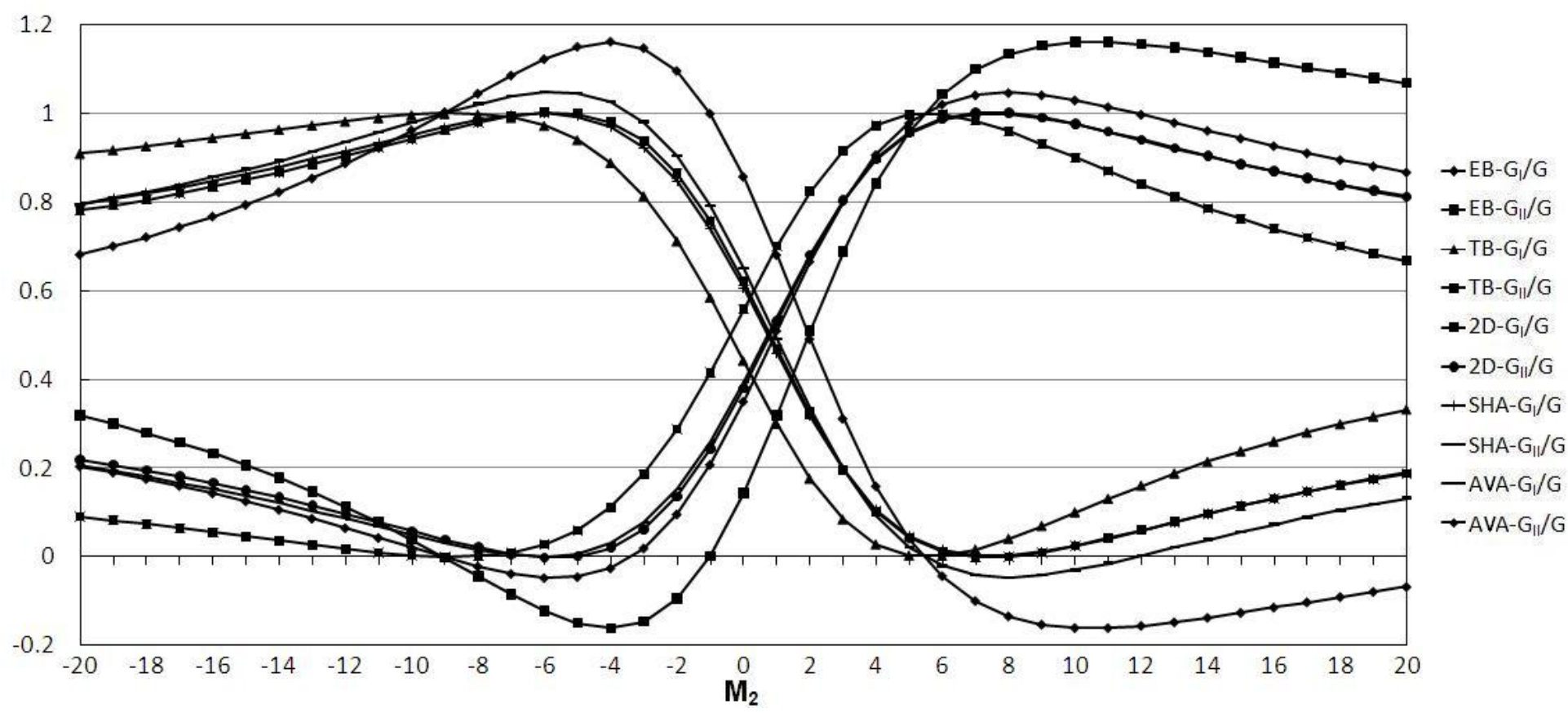

Figure 5: Fracture mode partitions in terms of $G_{I} / G$ and $G_{I I} / G$ for case $2, h_{1}+h_{2}=2, b=1$ and $\gamma=3$. 


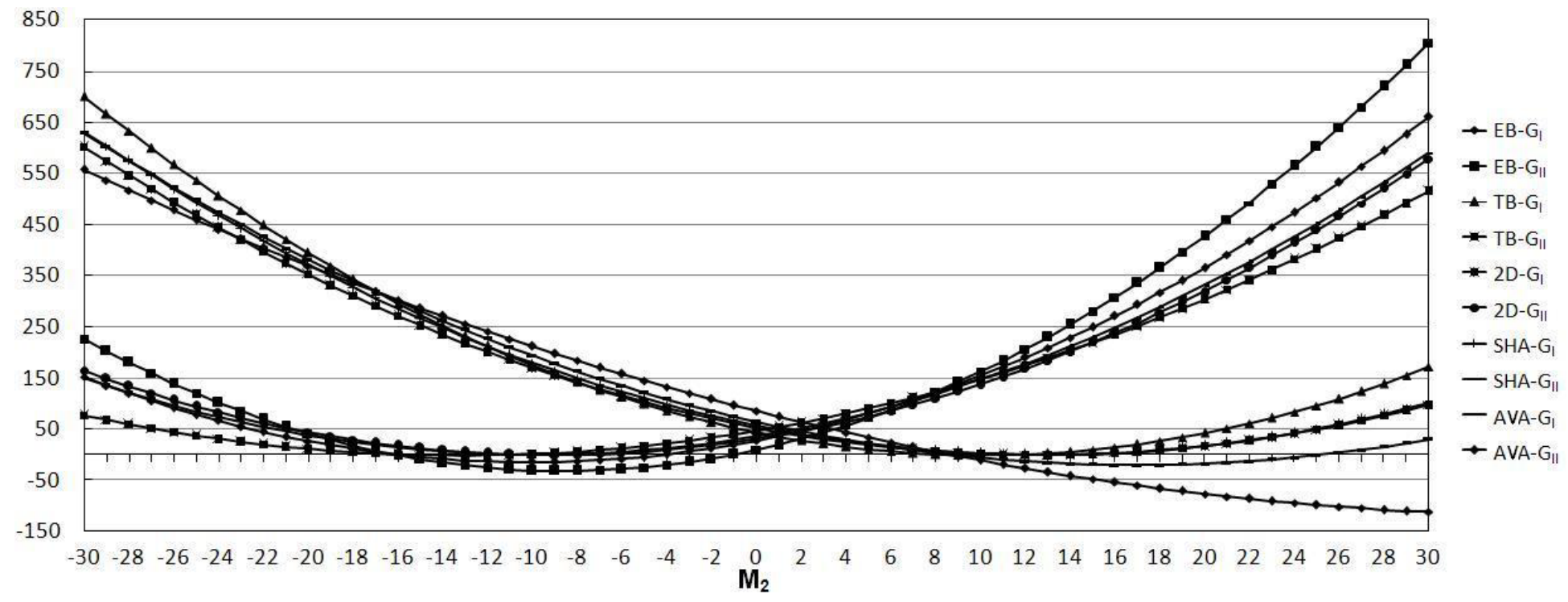

Figure 6: Fracture mode partitions in terms of $G_{I}$ and $G_{I I}$ for case $3, h_{1}+h_{2}=2, b=1$ and $\gamma=4$.

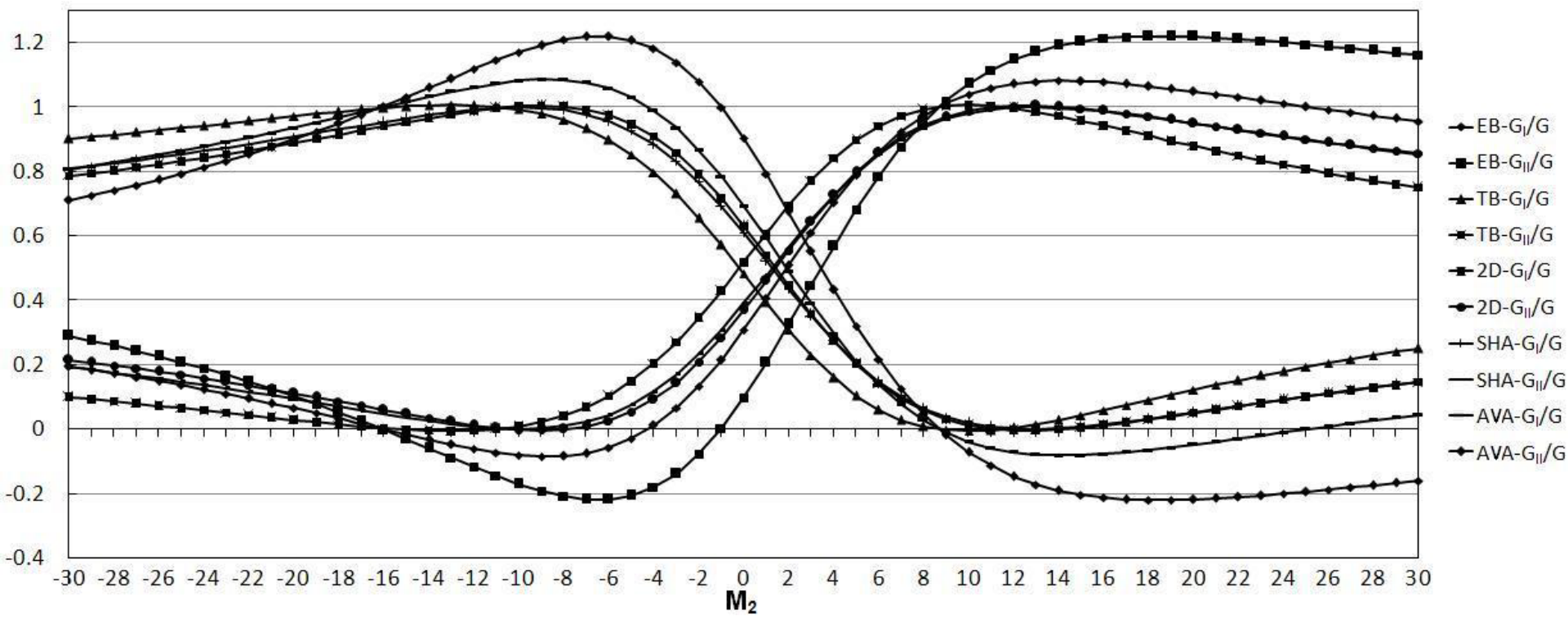

Figure 7: Fracture mode partitions in terms of $G_{I} / G$ and $G_{I I} / G$ for case $3, h_{1}+h_{2}=2, b=1$ and $\gamma=4$. 


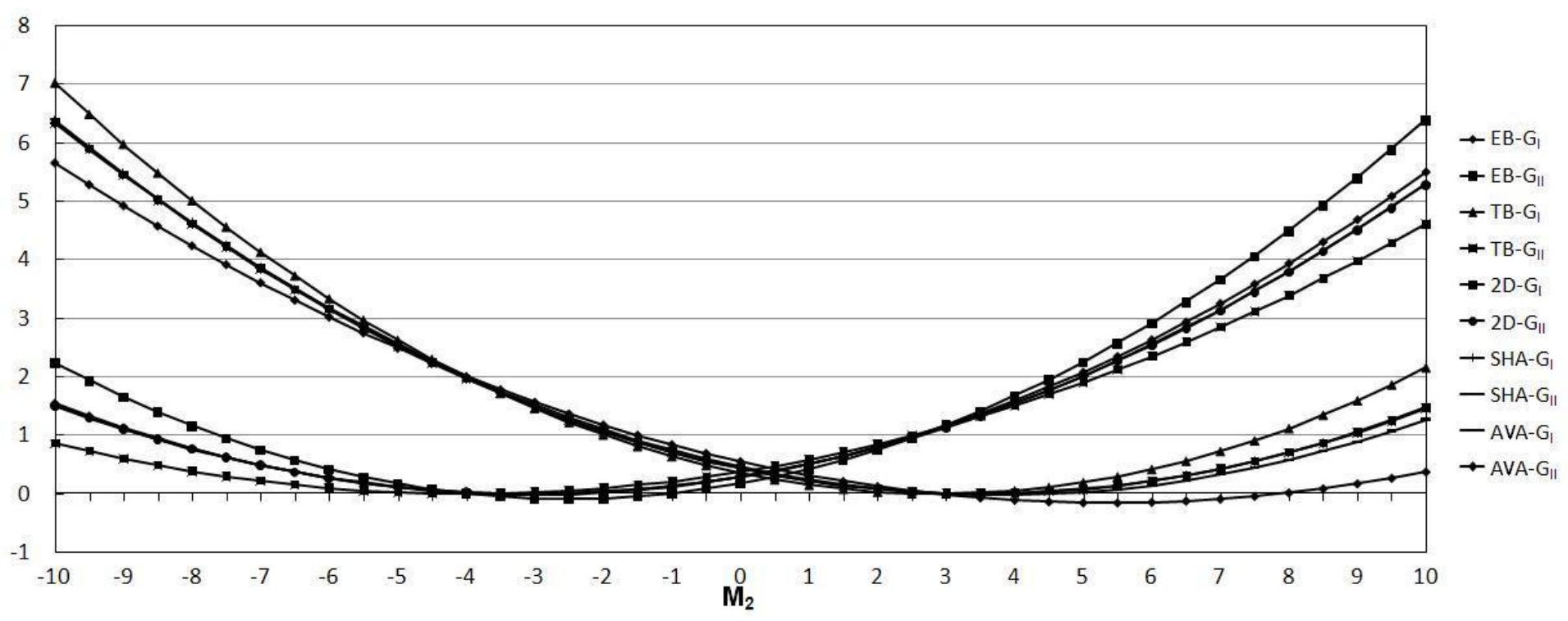

Figure 8: Fracture mode partitions in terms of $G_{I}$ and $G_{I I}$ for case $4, h_{1}+h_{2}=6, b=1$ and $\gamma=2$.

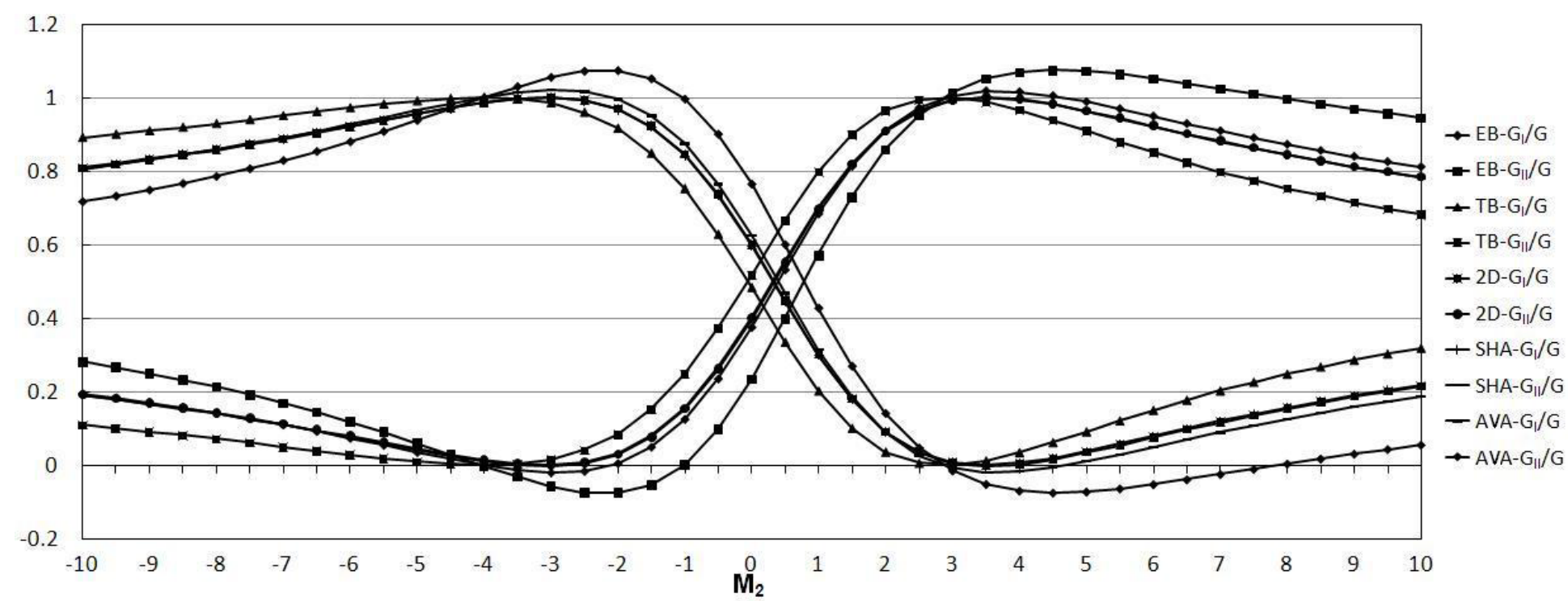

Figure 9: Fracture mode partitions in terms of $G_{I} / G$ and $G_{I I} / G$ for case $4, h_{1}+h_{2}=6, b=1$ and $\gamma=2$.

\section{Conclusions}

New partition theories are developed in the paper. Numerical investigations show that the present theoretical results based on Euler and Timoshenko beam theories are almost identical to their counterpart FEM predictions. Hutchinson and Suo theory [8] does not agree with either the present Euler or Timoshenko beam theory. However, it agrees very well with 2D FEM prediction. In general, the present averaged theory agrees well with the $2 \mathrm{D}$ plane stress FEM results in the non-negative $G_{I}$ and $G_{I I}$ regions. A distinct advantage of present theories is that they can be directly extended to fiber reinforced composite laminates for delamination study, which will be reported in future papers. Finally, it is noted that experimental works are required to validate the theories.

\section{References}

[1] S. Wang, C. Harvey, Fracture mode partition rules for DCB, 17th Interactional Conference on Composite/Nano Engineering

Honolulu, Hawaii, USA, July 2009.

[2] C. Harvey, S. Wang, Modelling of delamination propagation in composite laminated beam structures, Proceedings of the 7th International Conference of Computational 
Methods in Science and Engineering (ICCMSE 2009), T. E. Simos (ed), American Institute of Physics, Rhodes, Greece.

[3] S. Wang, C. Harvey, Mixed mode partition in one dimensional fracture, Periodical of Journal of Key Engineering Materials Vols. 462-463 (2011) 616-621. (Also, A plenary lecture in the 8th International Conference on Fracture and Strength of Solids (FEOFS 2010), Kuala Lumpur, Malaysia, June 2010).

[4] S. Wang, C. Harvey, Fracture mode partition theory for one dimensional fracture, presented at the 18th European Conference on Fracture (ECF18), Dresden, Germany, Aug. 30 - Sep. 03, 2010. [5] L. Guan, S. Wang, On Fracture Mode Partitions, presented at the 20th International
Workshop on Computational Mechanics of Materials, Loughborough University, UK, Sep. 2010.

[6] Y. Zhang, S. Wang, Buckling, post-buckling and delamination propagation in debonded composite laminates Part 1 Theoretical development, Composite Structures 88 (2009) 121-130.

[7] S. Wang, Y. Zhang, Buckling, post-buckling and delamination propagation in debonded composite laminates Part 2 Numerical applications, Composite Structures 88 (2009) 131-146.

[8] J. W. Hutchinson, Z. Suo, Mixed mode cracking in layered materials, Advances in Applied Mechanics, 29 (1992) 63-191. 